\title{
The Perfetti method, a novel Virtual Fine Motor Rehabilitation system for Chronic Acquired Brain Injury
}

\author{
Sergio Albiol-Pérez, \\ Guillermo Palacios- \\ Navarro \\ Universidad de Zaragoza \\ Ciudad Escolar s/n \\ 44003 - Teruel, SPAIN \\ +34978618102 \\ \{salbiol|g.palacios\} \\ @unizar.es
}

\author{
José-Antonio Gil-Gómez, \\ José-Antonio Lozano Quilis, \\ Hermenegildo Gil-Gómez \\ Universitat Politècnica de València, \\ C. Vera S/N, 46022, Valencia, \\ SPAIN \\ +34963877000
}

\{jgil | jlozano | hgil \}@upv.es
Pilar Manzano

Hospital S. José

Av. Zaragoza 16, 44001-Teruel, SPAIN +34978605368 pilarmanzanoh@ hotmail.com

\begin{abstract}
Acquired Brain Injury (ABI) is a disability with a high worldwide incidence that requires the assistance of a multidisciplinary team of clinic specialists. Motor disorders in patients of this type include dysfunctions in upper limbs, arm-hand impairments, spasticity, and functionalities that patients need to recover in order to perform the basic and instrumented activities of daily living. Traditional rehabilitation techniques in upper-limb rehabilitation for improving motor recovery are focused on repetitive and highintensity task-specific training. Promising new systems based on Virtual Fine Motor Rehabilitation (VFMR) are a novel approach in the rehabilitation process. In this paper, we describe a VFMR system (VPREHAB) for Chronic ABI patients to improve the effectiveness of upper-limb rehabilitation. For this purpose, we are testing the usefulness of the Perfetti method, a cognitive sensory motor therapy.
\end{abstract}

\section{Categories and Subject Descriptors}

H5.1. [Multimedia Information Systems]: Artificial, augmented, and virtual realities; H.5.2 [Information Interfaces and Presentation]: User Interfaces - Graphical user interfaces (GUI), Interaction styles, Screen design, User-centered design. J.3 [Computer Applications]: Life and medical Sciences - Health, Medical information systems.

\section{General Terms}

Design, Experimentation, Performance.

\section{Keywords}

Virtual Fine Motor Rehabilitation; Perfetti method; Acquired Brain Injury; Physical Therapy; Hand Rehabilitation; Arm Rehabilitation

\section{INTRODUCTION}

Acquired brain injury (ABI) is one of the leading causes of disability and death worldwide. The incidence of stroke in the

Permission to make digital or hard copies of all or part of this work for personal or classroom use is granted without fee provided that copies are not made or distributed for profit or commercial advantage and that copies bear this notice and the full citation on the first page. To copy otherwise, to republish, to post on servers or to redistribute to lists, requires prior specific permission and/or a fee. REHAB 2014, May 20-23, Oldenburg, Germany Copyright (c) 2014 ICST 978-1-63190-011-2 DOI 10.4108/icst.pervasivehealth.2014.255251
U.S. is around 795,000 every year (with an $87 \%$ ischemic, a $10 \%$ intracranial, and a 3\% subarachnoid hemorrhage). In 2008, approximately one out of 18 deaths was due to stroke [1]. The incidence of Traumatic Brain Injury (TBI) in the U.S. is approximately 1.74 million per year, with long-term disability from 3.32 million to 5.3 million, with mild severity of $80 \%$ [2].

Stroke is defined as a neurological disorder due to an acute injury of the central nervous system [3] that is produced by changes in the supply of blood to the brain [4]. These types of disorders are due to ischemic diseases (impairment or occlusion of an artery in the brain), intracerebral hemorrhage (ICH), and subarachnoid hemorrhage (SAH) [5].

Disability in TBI patients can be classified as primary or secondary. Primary damage is produced through external forces on the injured brain as a result of hits or penetration of objects caused by a blast [6]. Events of this type, which are influenced by the nature of the matter, intensity, direction, and length of external forces, determine the severity of the damage. Secondary damage is produced by bimolecular and physiological changes after the primary damage. This produces edemas, disturbances in brain functionality, increases in brain pressure, and reduction in blood flow [6]. Other types of secondary injuries are: 1) intracranial hematomas; 2) ischemia; 3) infection; 4) epilepsy; and 5) endocrine disturbances [7].

Clinical disturbances in ABI patients are related to loss of functionality in upper limbs, arm impairments, and spasticity [8], leading to dependence in their basic activities of daily living (ADL) and instrumented activities of daily living (iADL) [9]. Positive symptomatology of spasticity is composed of permanent contraction of the muscles, hypertonia (muscle tension with passive resistance of stress), stiffness, and contractions between agonist and antagonist muscles. Negative symptomatology is based on loss of skills and dexterity [10].

\section{RELATED WORK}

Due to these disturbances, traditional rehabilitation in upper limbs requires different assessments of specific repetitive tasks, specific movements, and position routine.

A Systematic Review was published in 2004 [11]. This review found that treatments of this type are carried out by occupational therapists and specialists in brain injury. The techniques used are the following: 1) constraint-induced movement therapy (CIMT) [12], which is a method that trains the affected upper limb by 
constraining the non-affected limb. However, this technique has the drawback that not all $\mathrm{ABI}$ patients can perform it due to the high severity of restriction in upper-limb movement; 2) repetitive and intensive upper-limb tasks, with significant improvement in neuromuscular, ADL, and functional outcomes [13]; 3) bilateral arm-training and dexterity of the paretic arm, with isometric and isotonic exercises in the affected arm (grip strength, isometric and isotonic hand extension etc.)[14]; 4) mirror therapy, moving the non-paretic arm while the ABI patient looks at a mirror and thinks that he/she is moving the paretic arm [15]. This type of therapy provides visual-feedback.

Few studies have validated the efficacy of Cognitive Sensory Motor Training Therapy (the Perfetti method) in the motor recovery of upper-limb ABI patients [16], but the outcomes are promising and encouraging.

However, these techniques do not maintain the interest of the patient who loses motivation. This leads to a decrease in the stimulation of working alliance and a reduction in the functional outcomes.

Currently, Virtual Motor Rehabilitation (VMR) that is carried out in the rehabilitation process of $\mathrm{ABI}$ patients is a promising approach. Different studies have tested and validated with effectiveness of VMR in balance, postural control, lowers limb, and uppers limb [17],[18],[19]. The use of customizable Virtual Environments (VE) with visual and auditory feedback produces comfortable training sessions, thereby improving the final results and outperforming traditional rehabilitation.

New systems using personal tablets or low-cost optical tracking devices (Ms. Kinect) are a novel discipline in the rehabilitation of ABI patients that provides sensory feedback to the brain [20],[21]. The drawback of these systems is the use of commercial games that are not suitable for virtual rehabilitation therapies, due to the complexity of the games. To obtain adequate motor recovery, it is necessary to develop specific and customizable systems that are focused on the rehabilitation of the upper limbs in chronic ABI patients.

The purpose of this study is to test gross/fine motor rehabilitation in chronic ABI patients using Perfetti method. To do this, we have designed a specific system, Virtual Perfetti Rehabilitation (VPREHAB), which is focused on hand movements training (hand opening/closing and flexion/extensions) and forearm rotation (pronation/supination and adduction/abduction of the wrist). By using VPREHAB, the therapeutic process will increase the recovery of the hand and arm in chronic ABI patients through highs levels of satisfaction and enjoyable rehabilitation sessions.

\section{METHODS}

\subsection{Participants}

The study is being conducted with chronic ABI patients. The inclusion criteria are: 1) age $\geq 18$ years and $\leq 70$ years; 2) chronicity $\geq 24$ months; 3) comprehension of VPREHAB instructions (the Mississippi Aphasia Screening Test (MAST) $\geq 45$ [22]); 4) baseline outcomes of the modified Ashworth Spasticity Scale (ASS) $<2$ and close to zero; 5) patients without cognitive impairment (MEC-Lobo [27]) > 23. The exclusion criteria are: 1) chronic ABI patients with visual/auditory injuries; 2) patients with hemispatial neglect; 3) traumatological injury that is not properly solved; 5) refusal of the chronic ABI patient.

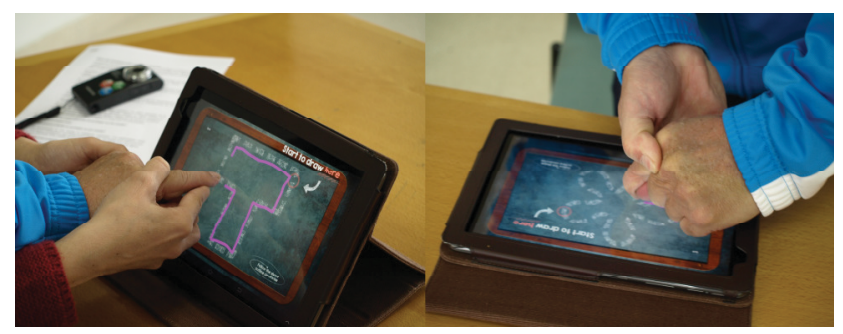

Figure 1. Subject using VPREHAB.

The sample consists of 20 chronic ABI patients (stroke and TBI patients), with residual paresis or plegia in upper limb.

In accordance with these clinical requirements, the designed system is composed of three main stages: selection of patient/games, game play, and results.

\subsection{VPREHAB System}

VPREHAB is composed of different Virtual Environments (VE) that have been designed and developed by a multidisciplinary team that is composed of therapists and clinical specialists in rehabilitation.

In our opinion, the goals that we want to achieve are: 1) an increase in the recovery of upper limbs for different pathologies in chronic ABI patients such as stroke, TBI, and others; 2) the use of a system that motivates chronic ABI patients; 3) the use of visual/auditory feedback that shows the results achieved in the therapeutic sessions; 4) improvements in upper limbs and muscle tone in chronic ABI patients; 5) the creation of a novel system to perform the virtual Perfetti method at home; 6) the customization of virtual sessions for each patient in this study.

The therapeutic exercises that patients carry out are based on abnormal reactions to stretching. These types of movements are included in the first level of the Perfetti method, where the perceptive hypothesis is the recognition of figures or letters of the alphabet (Figure 1).

The cognitive tasks that chronic $\mathrm{ABI}$ patients perform are composed of two phases: 1) the sensorimotor exploration phase, in which patients are sitting in front of a wood board (in the traditional Perfetti method session)/ or in from of a personal tablet (in the VPREHAB session). At this point, the patients are shown different figures or letters, with special attention to the outline of the shape of the objects; 2) the sensorimotor identification phase, in which patients close their eyes and the therapist places a part of the patient's hand guiding his/her fingertip with precise and uniform movements along the outline of the shape selected. In this phase, VPREHAB plays sound cues to reinforce the precise and controlled fingertip movements.

The exercises of this study are related to the repetition of flexion/extension movements with the collaboration of the therapist and the chronic ABI patients and to the perceptive task of the recognition of the angle that the fingers acquire. The different levels of difficulty are based on parameters such as an increase in the number of figures, the use of a greater number of fingers, and an increase in average speed.

For medium-level exercises, the assumed hypothesis for the first module is based on the recognition of the length of the different targets. In the sensorimotor identification phase, the patient closes 


\begin{tabular}{c} 
VPREHAB MENU \\
\hline \hline Trainning Difficulty Level
\end{tabular}

\begin{tabular}{cc}
\hline \hline \multicolumn{2}{c}{ FINE MOTOR MOVEMENTS } \\
\hline \hline Medium Level & Screenshot \\
\hline
\end{tabular}

The therapist helps the patient to move their fingertips by placing them on the screen on the different bars so that the patient can identify each one of them.

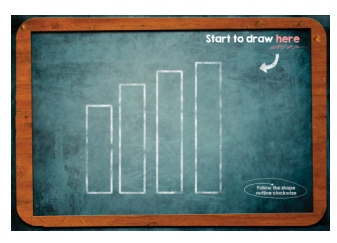

\begin{tabular}{cc}
\hline Medium Level & Screenshot \\
\hline
\end{tabular}

The patient (with the assistance of the therapist) moves four fingers (except the thumb) with a uniform movement that traces the selected trajectory. The patient should identify every single trajectory.

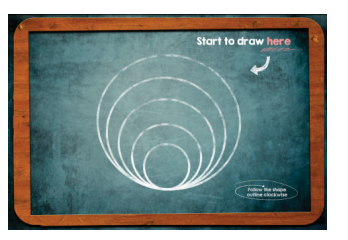

\begin{tabular}{cc}
\hline \hline High Level & Screenshot \\
\hline
\end{tabular}

The patient carries out movements that are harder to perform by guiding his/her fingers in a uniform way following one of the predetermined trajectories.

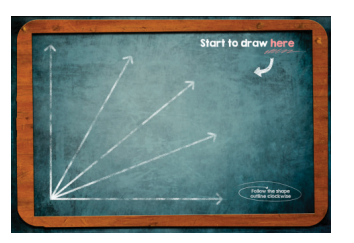

Table 1. VPREHAB Virtual Environtments.

his/her eyes and the VPREHAB system successively displays different bars (of different lengths and colors), and the patient has to identify each one of them. In this therapeutic exercise, the level of difficulty deals with the simultaneous use of a higher number of bars or a greater number of fingers. The hypothesis for the second module is based on space recognition by performing flexion/extension movements and adduction/abduction wrist movements.

For high-level exercises, the hypothesis is based on the optimization of trajectory drawing. The patients have to perform simple straight trajectories without the assistance of the therapist.

The different fine virtual motor movements based on cognitive tasks are shown in Table 1.

Other measures that we test are: 1) "Reaction time" (the time elapsed from when VPREHAB shows the Virtual Environment until the patient starts and touches the screen); 2) "Movement precision" (the accuracy with which the chronic ABI patient performs the task correctly); 3) "Completion time" (the total time that the chronic $\mathrm{ABI}$ patients need to perform the task using VPREHAB).

Finally, the system stores all the information related to the chronic ABI patients in every session in the cloud. This information allows the clinical specialists to obtain good feedback from the virtual rehabilitation process using VPREHAB, showing the improvements made by chronic ABI patients.

The originality of this contribution is based on: 1) the patientVPREHAB interaction, where the patient has to simulate gross/fine upper-limb movements; and 2) the visual/auditory feedback added by VPREHAB, which increases the adherence to the treatment and the motivation thanks to the playful component of our system. This aspect is barely treated in traditional rehabilitation.

\section{PROCEDURE}

The chronic ABI patients will perform a total of 20 sessions using VPREHAB of 3 to 5 sessions per week of approximately 30 minutes using VPREHAB and 30 minutes of traditional upperlimb rehabilitation. At the present time, we are testing different standardized measures for three periods of time (Initial, Final, and Follow-up Evaluation).

At recruitment, the chronic ABI patients' characteristics will be stored, and the therapist will perform different clinical tests that are related to Cognitive Sensory Motor training such as: the Action Research Arm test (ARAT) [23]; the Wolf Motor Function test (WMFT) [24]; the modified Ashworth Spasticity Scale (ASS) [25]; and the Box and block test (BBT)[26].

Another secondary clinical test that we will perform is an adapted and validated version of the Mini-Mental state examination [27].

At the end of the first session, we will use the Suitability Evaluation Questionnaire (SEQ) [28] to obtain a metric of usability of our Virtual Rehabilitation experiment, in order to determine whether the VPREHAB system complies with the standards of usability, acceptance, robustness, and validity.

\section{RESULTS}

At the present time, the study is a work in progress. The participants are Chronic ABI patients with mild to moderate spasticity, mild to moderate hypertonia, and that have sufficient muscle tone with, with minimal resistance in the range of flexion/extension motion of the paretic upper limb, and mean values in the ASS ranging from 0 to 2 , which is appropriate for the purposes of this study.

The results that we are currently obtaining are based on the patients' feedback and their high level of satisfaction. We consider that, in the near future, we will obtain promising results at the assessment stage.

\section{DISCUSSION AND CONCLUSIONS}

Currently, this study is showing promising and satisfactory benefits for ABI patients in the rehabilitation process. The goal of this study is to improve Cognitive Sensory Motor Training Therapy by means of the Perfetti method, using of a VFMR. To date, the chronic ABI patients who are participating in this study are showing great motivation during the training sessions. For this reason, we are encouraged to continue the treatment and to obtain 
high levels of functionality related to muscle tone, pronation/supination, flexion/extension, and adduction/abduction of the wrist. Future functionalities will be based on the design of new modules in the VPREHAB system to train other levels of the Perfetti method based on the pathology of chronic ABI patients.

\section{ACKNOWLEDGEMENTS}

The authors would like to acknowledge the work done by the specialist team in designing the Virtual Environments and Game Engines, especially Mr. Alejandro del Rio and Ms. Minerva Rodriguez.

This contribution was partially funded by the Gobierno de Aragón, Departamento de Industria e Innovación, y Fondo Social Europeo "Construyendo Europa desde Aragón" and by the Fundación Antonio Gargallo ("Ayudas financiadas por la Obra Social de Ibercaja de proyectos de investigación 2013", proyecto 2013/B001).

\section{REFERENCES}

[1] Roger V.L., Go A.S., Lloyd-Jones D.M., et al. 2012. American Heart Association Statistics Committee and Stroke Statistics Subcommittee. Heart disease and stroke statistics--2012 update: a report from the American Heart Association. Circulation, 125, e2e220.

[2] Ma V.Y., Chan L., Carruthers K.J. 2014. The Incidence, Prevalence, Costs and Impact on Disability of Common Conditions Requiring Rehabilitation in the US: Stroke, Spinal Cord Injury, Traumatic Brain Injury, Multiple Sclerosis, Osteoarthritis, Rheumatoid Arthritis, Limb Loss, and Back Pain. Arch Phys Med Rehabil, pii: S0003-9993, 00031-8.

[3] Sacco R.L., Kasner S.E., Broderick J.P., et al. 2013. An updated definition of stroke for the 21st century: a statement for healthcare professionals from the American Heart Association/American Stroke Association. Stroke, 44, 2064-89.

[4] Donnan G.A., Fisher M., Macleod M., Davis S.M. 2008. Stroke. Lancet, 371, 1612-23.

[5] Sehba F.A., Pluta R.M., Macdonald R.L. 2013.Brain injury after transient global cerebral ischemia and subarachnoid hemorrhage. Stroke Res Treat, 827154 .

[6] Greve M.W., Zink B.J. 2009. Pathophysiology of traumatic brain injury. Mount Sinai Journal of Medicine, 76, 97-104.

[7] Pushkarna A., Bhatoe H.S., Sudambrekar S.M. 2010. Head injuries. Medical Journal Armed Forces India, 66, 321-324.

[8] Leonard C.T., Gardipee K.A., Koontz J.R., Anderson J.H., Wilkins S.A. 2006. Correlation between impairment and motor performance during reaching tasks in subjects with spastic hemiparesis. J Rehabil Med, 38, 243-9.

[9] Graf C., Hartford Institute for Geriatric Nursing. The Lawton instrumental activities of daily living (IADL) scale. Medsurg Nurs, 17, 343-4.

[10] Mayer N.H., Esquenazi A., Childers M.K. 1997. Common patterns of clinical motor dysfunction. Muscle Nerve Suppl, 6, 21-35.

[11] Van Peppen R.P., Kwakkel G., Wood-Dauphinee S., Hendriks H.J., Van der Wees P.J., Dekker J. 2004. The impact of physical therapy on functional outcomes after stroke: what's the evidence?. Clin Rehabil,18, 833-62.

[12] Dettmers C., Teske U., Hamzei F., Uswatte G., Taub E., Weiller C. 2005. Distributed form of constraint-induced movement therapy improves functional outcome and quality of life after stroke. Arch Phys Med Rehabil, 86, 204-9.
[13] Kwakkel G., Wagenaar R.C., Koelman T.W., Lankhorst G.J., Koetsier J.C. 1997. Effects of intensity of rehabilitation after stroke. A research synthesis. Stroke. 28, 1550-6.

[14] Luft A.R., McCombe-Waller S., Whitall J., Forrester L.W., Macko R., Sorkin J.D., et al. 2004. Repetitive bilateral arm training and motor cortex activation in chronic stroke: a randomized controlled trial. JAMA, 292, 1853-61. Erratum in: JAMA, 292(20), 2470, 2004.

[15] Bhasin A. Padma Srivastava M.V., Kumaran S.S., Bhatia R., Mohanty S. 2012. Neural interface of mirror therapy in chronic stroke patients: a functional magnetic resonance imaging study. Neurol India, 60, 570-6.

[16] Chanubol R., Wongphaet P., Chavanich N., Werner C., Hesse S., Bardeleben A., Merholz J. 2012. A randomized controlled trial of Cognitive Sensory Motor Training Therapy on the recovery of arm function in acute stroke patients. Clin Rehabil, 26, 1096-104.

[17] Gil-Gómez J.A., Lloréns R., Alcañiz M., Colomer C. 2011. Effectiveness of a Wii balance board-based system (eBaViR) for balance rehabilitation: a pilot randomized clinical trial in patients with acquired brain injury. J Neuroeng Rehabil, 23.

[18] Albiol-Pérez S., Gil-Gómez J.A., Llorens R., Alcañiz M., Font C.C. 2014. The role of virtual motor rehabilitation: a quantitative analysis between acute and chronic patients with acquired brain injury. IEEE $J$ Biomed Health Inform, 18, 391-8.

[19] Albiol-Perez S., Gil-Gomez J.A., Forcano-García M., Muñoz-Tomas M.T., Manzano-Hernandez P., Solsona-Hernandez S. 2013. Development of a novel therapy in virtual motor rehabilitation after severe Guillain-Barré symptomatology. Pervasive Computing Technologies for Healthcare (PervasiveHealth '13), 370-373.

[20] Lee G. 2013. Effects of training using video games on the muscle strength, muscle tone, and activities of daily living of chronic stroke patients. J Phys Ther Sci, 25, 595-7.

[21] Sin H., Lee G. 2013. Additional virtual reality training using Xbox Kinect in stroke survivors with hemiplegia. Am J Phys Med Rehabil. 92, 871-80.

[22] Nakase-Thompson R., Manning E., Sherer M., Yablon S.A., Gontkovsky S.L., Vickery C. 2005.Brief assessment of severe language impairments: initial validation of the Mississippi aphasia screening test. Brain Inj. 19, 685-691.

[23] Lyle R.C. 1981. A performance test for assessment of upper limb function in physical rehabilitation treatment and research. Int $J$ Rehabil Res, 4, 483-92.

[24] Wolf S.L., Catlin P.A., Ellis M., Archer A.L., Morgan B., Piacentino A. 2001. Assessing the Wolf motor function test as outcome measure for research in patients after stroke. Stroke, 32, 1635-9.

[25] Bohannon R.W., Smith M.B. 1987. Interrater reliability of a modified Ashworth scale of muscle spasticity. Phys Ther, 67, 206-7.

[26] Mathiowetz V., Volland G., Kashman N., Weber K. 1985. Adult norms for the Box and Block Test of manual dexterity. Am J Occup Ther, 39, 386-91.

[27] Folstein M.F., Folstein S.E., Mchugh P.R. 1975. Mini-Mental State: a practical method for grading the cognitive state of patients for the clinicians. J Psychiatr Res, 12, 189-198.

[28] Gil-Gómez J.A., Manzano-Hernández P., Albiol-Pérez S., AulaValero C., Gil-Gómez H., Lozano-Quilis J.A. 2013. SEQ: suitability evaluation questionnaire for virtual rehabilitation systems. Application in a virtual rehabilitation system for balance rehabilitation. Pervasive Computing Technologies for Healthcare (PervasiveHealth '13), 335-338, 2013. 